\title{
Cancer Mortality Among Brazilian Dentists
}

\author{
Sergio Koifman, $\mathrm{PhD}^{1}{ }^{1}$ Thainá Alves Malhão, $\mathrm{MSPH}^{2}{ }^{2}$ Gisele Pinto de Oliveira, $\mathrm{MSPH}^{2}$ \\ Volney de Magalhães Câmara, $\mathrm{PhD}^{2}{ }^{2}$ Rosalina Jorge Koifman, $\mathrm{PhD}^{1}{ }^{1}$ and Armando Meyer, $\mathrm{PhD}^{2}$
}

\begin{abstract}
Background Previous studies have variably shown excess risks of elected cancers among dentists.

Methods National Brazilian mortality data were used to obtain mortality patterns among dentists between 1996 and 2004. Cancer mortality odds ratios (MORs) and cancer proportional mortality ratios for all cancer sites were calculated, using the general population and physicians and lawyers as comparison groups.

Results Female dentists from both age strata showed higher risks for breast, colonrectum, lung, brain, and non-Hodgkin lymphoma. Compared to physicians and lawyers, higher MOR estimates were observed for brain cancer among female dentists 20-49 yr. Among male dentists, higher cancer mortality was found for colon-rectum, pancreas, lung, melanoma, and non-Hodgkin lymphoma. Higher risk estimates for liver, prostate, bladder, brain, multiple myeloma and leukemia were observed among 50-79 yr old male dentists. Discussion If confirmed, these results indicate the need for limiting occupational exposures among dentists in addition to establishing screening programs to achieve early detection of selected malignant tumors. Am. J. Ind. Med. 57:1255-1264, 2014.

(C) 2014 Wiley Periodicals, Inc.Am.
\end{abstract}

KEY WORDS: cancer; dentistry; mortality; occupational risks

\section{INTRODUCTION}

Dentists are exposed to diverse physical, biological, and chemical occupational hazards that may lead to adverse health outcomes [Szymanska, 1999; Al-Khatib et al., 2006; Simning and van Wijngaarden, 2007]. As a consequence, cancer risk among dentists has received considerable attention. Such studies have generally not found increases in all-site cancer mortality and incidence, but many have reported an increased risk for specific types of cancer among

${ }^{1}$ Department of Epidemiology and Quantitative Methods, National School of Public Health, Oswaldo Cruz Foundation, Rio de Janeiro, Brazil

${ }^{2}$ Institute for Studies in Collective Health, Federal University of Rio de Janeiro, Rio de Janeiro, Brazil

${ }^{*}$ Correspondence to: Armando Meyer, PhD, IESC/UFRJ Av. Horacio Macedo, S/N. Cidade Universitária. Rio de Janeiro - RJ. Brazil. CEP-21941-598.

E-mail:armando@iesc.ufrj.br

Accepted 10 June 2014

D0I 10.1002/ajim.22369. Published online 27 August 2014 in Wiley Online Library (wileyonlinelibrary.com). dentists, such as bladder [Blair et al., 1985], brain [Ahlbom et al., 1986; Preston-Martin, 1989; Vagero et al., 1990; Linet et al., 1995; Eriksson et al., 1998; Pollan and Gustavsson, 1999; Carozza et al., 2000; Navas-Acien et al., 2002; Krishnan et al., 2003; Antunes et al., 2004; Simning and van Wijngaarden, 2007], intestine [Blair et al., 1985], leukemia [Morton and Marjanovic, 1984; Blair et al., 1985], liver [Pollan and Gustavsson, 1999], lung [Blair et al., 1985], malignant lymphomas [Blair et al., 1985], skin [Vagero et al., 1990; Linet et al., 1995; Rix and Lynge, 1996; Eriksson et al., 1998; Pollan and Gustavsson, 1999; Antunes et al., 2004; Simning and van Wijngaarden, 2007], stomach [Blair et al., 1985], thyroid [Wingren et al., 1997; Eriksson et al., 1998; Antunes et al., 2004], melanoma [Perez-Gomez et al., 2004], tongue [Ji and Hemminki, 2005], and some cancers of reproductive-related organs and tissues [Simning and van Wijngaarden, 2007], especially female breast cancer [Blair et al., 1985; Sankila et al., 1990; Rix and Lynge, 1996; Rix et al., 1997; Krstev et al., 1998; Eriksson et al., 1998; Pollan and Gustavsson, 1999; Antunes et al., 2004; Simning and van Wijngaarden, 2007]. Other studies have not 
confirmed excess cancer among dentists [Hostettler and Minder, 2002; Nishio et al., 2004; Tanaka et al., 2004].

The present investigation was undertaken to ascertain the cancer mortality profile observed among Brazilian dentists during 1996-2004 and to compare it to the risk experienced by other occupational groups and the general population in Brazil.

\section{METHODS}

Data on cancer mortality among dentists, physicians, lawyers, and the general population in Brazil, were obtained from the National Mortality System. This system, which was implemented by the Ministry of Health, provides universal coverage and classifies causes of death according to the International Classification of Diseases (ICD) and has used the 10th edition since 1996. However, a study in 2000 that leukemia the cause of death of 618 individuals, in 15 counties, from 3 Brazilian states in different regions, revealed that 294 individuals $(47.6 \%)$ showed ill-defined causes of death [Jorge et al., 2002]. This result highlights the need for caution when analyzing mortality data in Brazil during the study period covered in this investigation.

Information was summarized on each reported death that occurred between 1996 and 2004 among 20-79 yr. old dentists (occupation code 063) that included a malignant neoplasm (ICD-10; C00-C97) as the primary cause of death in the death certificate. The dentists' cancer mortality for specific anatomical sites stratified by gender and age was compared with that experienced by the general population in Brazil. This rate was also compared with that among physicians and lawyers, both of whom have socio-economical levels quite similar to that of dentists. In this study, the mean monthly incomes of those careers in Brazil in the early twenty-first century were ascertained in reais (R): $\mathrm{R} \$ 3,031$ for dentists, R\$ 3,009 for lawyers, and R\$ 7,029 for physicians (Wikipedia, 2014).

Cancer mortality rates between dentists and the chosen reference groups were compared using mortality odds ratios (MORs), as suggested by Miettinen and Wang (1981). MOR is the ratio of the odds of mortality between the disease of interest (for instance, leukemia) and all other causes of death for a group of interest (i.e., dentists) compared to a reference group (such as physicians, lawyers, or all occupational groups in the general population), as carried out in this investigation [Wang and Miettinen, 1984; Meyer et al., 2003]. Hence, the conditions justifying the use of the proportionate mortality ratio (PMR) to compare exposed and unexposed populations also allow for the use of the MOR to compare a cause of death of interest with the other causes of death [Miettinen and Wang, 1981].

As indicated in the literature [Stewart and Hunting, 1988], the MOR can be overestimated when there is a healthy worker effect (HWE). In cases where the HWE is low, the authors indicated that the standardized mortality ratio (SMR) would range between the PMR (lower level) and the MOR (upper level), and the calculation of both statistics was suggested. Considering the heterogeneous quality of mortality data still prevailing in different regions of Brazil during the study period, the cancer proportional mortality ratio (CPMR), instead of the PMR, was ascertained. This choice was based on the high quality estimates of cancer mortality data that have been reported in Brazil since the 1990's [Monteiro et al., 1997].

In this study, the MOR was stratified according to gender and age at death (20-49 yr. and 50-79 yr.). Cancer deaths were compared with all other causes of death in the studied populations. Therefore, the causes of death among the controls were not confined to causes that are unrelated to the exposure. Hence, the ascertained risk estimates presented here may be lower than would occur if such a procedure were adopted.

For those cancer sites in which a higher MOR among dentists was observed, a sensitivity analysis was also performed following the exclusion of the reported major causes of deaths (cardiovascular diseases or external causes) among either physicians or lawyers.

Additionally, cancer proportional mortality ratios (CPMRs) were also ascertained for each cancer site according to gender and age. At first, the proportion of deaths by a specific primary site according to gender and age in the general population, or a specific occupational group (for instance, lawyers and physicians), was obtained relative to the total number of cancer deaths for the same gender and age stratum. Furthermore, such proportions were used to estimate the number of expected deaths that the interest group (dentists) would experience if they had the same cancer mortality pattern as that of the comparison group (the general population, physicians or lawyers). Finally, CPMRs were obtained according to age and gender that reflected the observed amount of cancer deaths and those expected at such specific primary sites.

\section{Ethical Aspects}

The Ethics Committee of the Institute for Studies in Collective Health, Federal University of Rio de Janeiro, approved the research protocol.

\section{RESULTS}

Overall, 3,583 deaths among dentists 20-79 yr old were reported by the Brazilian National Mortality System from 1996 to 2004. The main observed causes of death among the dentists were cardiovascular diseases (ICD-10: I00-I99, 1,113 deaths, $31.1 \%$ ), followed by cancer (ICD-10: C00D48, 988 deaths, 27.6\%) and external causes (ICD-10: V01- 
Y98, 490 deaths, 13.7\%). The distribution of all causes of death by age and gender in the general population, dentists, physicians, and lawyers is presented in Supplemental Table S1. The sensitivity analyses that were performed for both gender and age strata, following the exclusion of controls with cardiovascular and external causes of death, are in Supplemental Tables S2-S9.

\section{Cancer Mortality Among Brazilian Dentists}

\section{Male dentists 20-49 yr}

Compared to the general population, the overall mortality odds ratio (MOR) in male dentists ages 20-49 yr. was 4.29 (95\% C.I. 3.42-5.47) (Table I). The analysis of soft tissue sarcoma mortality among dentists revealed a MOR = 8.87 (95\% C.I. $2.41-22.7)$ and a CPMR $=4.10(95 \%$ C.I. 1.11-10.5). Similar comparisons performed with physicians and lawyers as the reference groups reported the following estimates: $\mathrm{MOR}=4.25$ (95\% C.I. 1.16-10.9) and CPMR $=$ 5.11 (95\% C.I. 4.08-6.39) compared to physicians, and $\mathrm{MOR}=7.34 \quad(95 \%$ C.I. $2.00-18.8)$ and $\mathrm{CPMR}=6.46$ (95\% C.I. 5.15-8.01) compared to lawyers.

\section{Male dentists 50-79 yr}

Compared to the general population, the all cancer mortality rate among male dentists ages $50-79$ yr. (Table II) showed a MOR $=1.67$ (95\% C.I. $1.54-1.81)$. When considering only multiple myeloma, a cancer $\mathrm{MOR}=3.55$ $(95 \%$ C.I. $2.07-5.68)$ and a CPMR $=2.36(95 \%$ C.I. $1.38-$ $3.78)$ were observed compared to the general population. Compared to lawyers, the observed risk estimates were an $\mathrm{MOR}=1.74(95 \%$ C.I. $1.01-2.78)$ and a $\mathrm{CPMR}=1.73$ (95\% C.I. 1.59-1.87).

Increased MORs and CPMRs were observed among male dentists compared to the general population, but not relative to male physicians and lawyers (Table II), for the following cancers: pancreas, $\mathrm{MOR}=2.66(95 \%$ C.I. $1.90-3.62)$ and $\mathrm{CPMR}=1.76$ (95\% C.I. 1.18-2.39); lung, $\mathrm{MOR}=1.80$ $(95 \%$ C.I. $1.51-2.15)$ and CPMR $=1.17$ (95\% C.I. $0.98-$ 1.39); non-Hodgkin lymphoma, MOR $=2.54$ (95\% C.I. 1.55 3.91) and $\mathrm{CPMR}=1.68$ (95\% C.I. 1.03-2.59); multiple myeloma, $\mathrm{MOR}=3.55$ (95\% C.I. 2.07-5.68) and CPMR $=$ 2.36 (95\% C.I. 1.38-3.78); and leukemia, $\mathrm{MOR}=3.30$ $(95 \%$ C.I. $2.21-4.75)$ and CPMR $=2.18(95 \%$ C.I. $1.46 ; 3.14)$.

\section{Female dentists 20-49 yr}

Compared to the general population, female dentists aged 20-49 yr (Table III) showed an all-site cancer $\mathrm{MOR}=2.26$ (95\% C.I. 1.91-2.67). When female dentists were compared to physicians and lawyers, such risk estimates were a $\mathrm{MOR}=1.35(95 \%$ C.I. $1.14-1.60)$ and $\mathrm{aOR}=1.38$ (95\% C.I. 1.17-1.63), respectively.

Breast cancer mortality risk estimates among female dentists ages $20-49$ yr. revealed a $\mathrm{MOR}=3.67(95 \%$ C.I.

TABLE I. Cancer Mortality Risk, Male Dentists Comparatively to Male Physicians and Lawyers, 20-49 yr, Brazil, 1996-2004.

\begin{tabular}{|c|c|c|c|c|c|c|c|c|c|c|}
\hline Death causes (ICD10*) & n Dent & n Gen Pop & MOR 95\%CI & CPMR & n Phys & MOR 95\%CI & CPMR & n Law & MOR 95\%CI & CPMR \\
\hline C00-C97 All Malignant neoplasms & 79 & 82,264 & $4.29(3.42-5.47)$ & & 202 & $0.80(0.64-1.00)$ & & 255 & $1.15(0.92-1.44)$ & \\
\hline C00-C14 Oral Cavity & 01 & 7,205 & $0.31(0.01-1.73)$ & $0.14(0.00-0.78)$ & 03 & $0.70(0.02-3.90)$ & $0.85(0.68-1.07)$ & 10 & $0.36(0.01-2.01)$ & $0.32(0.26-0.40)$ \\
\hline C15 Esophagus & 01 & 5,731 & $0.39(0.01-2.17)$ & $0.18(0.00-1.00)$ & 02 & $1.06(0.03-5.90)$ & $1.28(1.02-1.60)$ & 03 & $1.22(0.03-6.80)$ & $1.08(0.86-1.34)$ \\
\hline C16 Stomach & 02 & 8,039 & $0.56(0.07-2.02)$ & $0.26(0.03-0.94)$ & 17 & $0.25(0.03-0.90)$ & $0.30(0.24-0.38)$ & 14 & $0.52(0.06-1.88)$ & $0.46(0.37-0.58)$ \\
\hline C17 Small intestine & 02 & 256 & $17.5(2.12-63.3)$ & $8.13(0.98-29.3)$ & 00 & - & - & 01 & $7.32(0.89-26.43)$ & $6.46(5.15-8.07)$ \\
\hline C18-21 Colon-rectum & 07 & 4,881 & $3.24(1.30-6.67)$ & $1.49(0.60-3.07)$ & 25 & $0.59(0.24-1.22)$ & $0.72(0.57-0.89)$ & 30 & $0.85(0.34-1.75)$ & $0.75(0.60-0.94)$ \\
\hline C22 Liver and intrahepatic bile ducts & 03 & 3,613 & $1.86(0.38-5.43)$ & $0.86(0.18-2.51)$ & 10 & $0.63(0.13-1.84)$ & $0.77(0.61-0.96)$ & 15 & $0.73(0.15-2.13)$ & $0.65(0.52-0.81)$ \\
\hline C25 Pancreas & 05 & 2,437 & $4.62(1.50-10.8)$ & $2.14(0.69-4.99)$ & 07 & $1.51(0.49-3.52)$ & $1.83(1.46-2.28)$ & 07 & $2.62(0.85-6.10)$ & $2.31(1.84-2.88)$ \\
\hline C32 Larynx & 01 & 3,228 & $0.69(0.02-3.84)$ & $0.32(0.01-1.78)$ & 2 & $1.06(0.03-5.90)$ & $1.28(1.02-1.60)$ & 03 & $1.22(0.03-6.80)$ & $1.08(0.86-1.34)$ \\
\hline C34 Lung & 12 & 8,147 & $3.34(1.73-5.85)$ & $1.53(0.79-2.68)$ & 31 & $0.81(0.42-1.42)$ & $0.99(0.79-1.24)$ & 39 & $1.13(0.58-1.98)$ & $0.99(0.79-1.24)$ \\
\hline C38 Heart, mediastinum and pleura & 01 & 569 & $3.94(0.10-21.9)$ & $1.83(0.05-10.2)$ & 00 & - & - & 05 & $0.73(0.02-4.07)$ & $0.65(0.52-0.81)$ \\
\hline C40-41 Bone and articular cartilage & 03 & 1,533 & $4.40(0.91-12.8)$ & $2.04(0.40-5.96)$ & 03 & $2.12(0.44-6.19)$ & $2.56(2.04-3.20)$ & 02 & $5.50(1.13-16.06)$ & $4.84(3.86-6.05)$ \\
\hline C43-C44 Skin & 07 & 2,208 & $7.17(2.88-14.8)$ & $3.30(1.32-6.80)$ & 08 & $1.86(0.75-3.83)$ & $2.24(1.79-2.80)$ & 16 & $1.60(0.64-3.30)$ & $1.41(1.13-1.77)$ \\
\hline C43 Malignant melanoma of skin & 07 & 1,474 & $10.7(4.31-22.1)$ & $4.94(1.98-9.88)$ & 02 & $2.13(0.85-4.39)$ & $2.56(2.04-3.20)$ & 14 & $1.83(0.73-3.77)$ & $1.61(1.29-2.02)$ \\
\hline C44 Other malignant neoplasms of skin & 00 & 734 & - & 0.00 & 01 & - & - & 2 & - & - \\
\hline C49 Soft tissues & 04 & 1,015 & $8.87(2.41-22.7)$ & $4.10(1.11-10.5)$ & 02 & $4.25(1.16-10.88)$ & $5.11(4.08-6.39)$ & 2 & $7.34(2.00-18.79)$ & $6.46(5.15-8.07)$ \\
\hline C61 Prostate & 01 & 585 & $3.83(0.10-21.3)$ & $1.78(0.04-9.91)$ & 03 & $0.70(0.02-3.90)$ & $0.85(0.68-1.07)$ & 3 & $1.22(0.03-6.80)$ & $1.08(0.86-1.34)$ \\
\hline C71Brain & 05 & 6,611 & $1.70(0.55-3.96)$ & $0.79(0.26-1.84)$ & 23 & $0.45(0.15-1.05)$ & $0.56(0.44-0.69)$ & 18 & $1.01(0.33-2.35)$ & $0.90(0.72-1.12)$ \\
\hline C73-C75 Thyroid and other endocrine glands & 02 & 551 & $8.15(0.99-29.4)$ & $3.78(0.46-13.6)$ & 01 & $4.24(0.51-15.31)$ & $5.11(4.08-6.39)$ & 01 & $7.32(0.89-26.43)$ & $6.46(5.15-8.07)$ \\
\hline C81Hodgkin's disease & 02 & 1,045 & $4.29(0.52-15.5)$ & $1.99(0.24-9.17)$ & 03 & $1.41(0.17-5.09)$ & $1.70(1.36-2.13)$ & 03 & $2.44(0.30-8.81)$ & $2.15(1.72-2.69)$ \\
\hline C82-C85 Non-Hodgkin's lymphoma & 06 & 3,644 & $3.71(1.36-8.09)$ & $1.71(0.63-3.73)$ & 09 & $1.41(0.52-3.07)$ & $1.70(1.36-2.13)$ & 16 & $1.37(0.50-2.99)$ & $1.21(0.97-1.51)$ \\
\hline C91-C95 Leukemia & 05 & 6,025 & $1.86(0.60-4.33)$ & $0.86(0.28-2.00)$ & 17 & $0.62(0.20-1.44)$ & $0.75(0.60-0.94)$ & 19 & $0.96(0.31-2.24)$ & $0.85(0.68-1.06)$ \\
\hline
\end{tabular}

$n$ Dent $=$ number of deaths among dentists; $n$ Phys = number of deaths among physicians; $n$ Law = number of deaths among lawyers. 
TABLE II. Cancer Mortality Risk, Male Dentists Comparatively to Male Physicians and Lawyers, 50-79 yr, Brazil, 1996-2004.

\begin{tabular}{|c|c|c|c|c|c|c|c|c|c|c|}
\hline \multirow[b]{2}{*}{ Death causes (ICD10) } & \multirow[b]{2}{*}{ n Dent } & \multicolumn{3}{|c|}{ MOR } & \multicolumn{3}{|c|}{ MOR } & \multicolumn{3}{|c|}{ MOR } \\
\hline & & n Gen Pop & $(95 \% \mathrm{Cl})$ & CPMR & n Phys & $(95 \%$ CI) & CPMR & n Law & (C195\%) & CPMR \\
\hline C00-C97 All Malignant neoplasms & 604 & 403,688 & $1.67(1.54-1.81)$ & & 1226 & $0.89(0.82-0.96)$ & & 2578 & $1.01(0.93-1.09)$ & \\
\hline C00-C14 Oral cavity & 14 & 24,386 & $0.57(0.31-0.96)$ & $0.38(0.21-0.64)$ & 24 & $1.08(0.59-1.81)$ & $1.18(1.09-1.28)$ & 74 & $0.81(0.44-1.36)$ & $0.81(0.74-0.87)$ \\
\hline C15 Esophagus & 22 & 28,887 & $0.76(0.48-1.15)$ & $0.51(0.33-0.77)$ & 24 & $1.71(1.07-2.58)$ & $1.86(1.71-2.01)$ & 65 & $1.46(0.92-2.20)$ & $1.44(1.33-1.56)$ \\
\hline C16 Stomach & 28 & 48,294 & $0.57(0.38-0.83)$ & $0.38(0.25-0.54)$ & 69 & $0.75(0.50-1.09)$ & $0.82(0.76-0.89)$ & 154 & $0.78(0.52-1.13)$ & $0.78(0.71-0.84)$ \\
\hline C17 Small intestine & 03 & 1,064 & $2.83(0.58-8.26)$ & $1.88(0.39-5.49)$ & 03 & $1.86(0.38-5.43)$ & $2.03(1.87-2.19)$ & 09 & $1.43(0.29-4.18)$ & $1.42(1.31-1.54)$ \\
\hline C18-21 Colon-rectum & 54 & 22,863 & $2.40(1.81-3.15)$ & $1.58(1.20-2.07)$ & 137 & $0.73(0.55-0.96)$ & $0.80(0.74-0.86)$ & 270 & $0.85(0.64-1.12)$ & $0.85(0.79-0.92)$ \\
\hline C22 Liver & 26 & 17,657 & $1.48(0.97-2.18)$ & $0.98(0.64-1.44)$ & 57 & $0.85(0.56-1.25)$ & $0.93(0.85-1.00)$ & 134 & $0.83(0.54-1.22)$ & $0.83(0.76-0.89)$ \\
\hline $\begin{array}{l}\text { C23-C24 Gallbladder and other } \\
\text { unspecified parts of biliary tract }\end{array}$ & 06 & 4,386 & $1.37(0.50-2.99)$ & $0.91(0.33-1.98)$ & 15 & $0.74(0.27-1.61)$ & $0.81(0.75-0.88)$ & 40 & $0.64(0.23-1.40)$ & $0.64(0.59-0.69)$ \\
\hline C25 Pancreas & 40 & 15,202 & $2.66(1.90-3.62)$ & $1.76(1.18-2.39)$ & 83 & $0.89(0.64-1.21)$ & $0.98(0.90-1.06)$ & 166 & $1.03(0.74-1.40)$ & $1.03(0.95-1.11)$ \\
\hline $\begin{array}{l}\text { C30-31 Nasal cavity. middle ear and } \\
\text { accessory sinuses }\end{array}$ & 01 & 520 & $1.93(0.05-10.7)$ & $1.28(0.03-1.56)$ & 00 & & - & 01 & $4.29(0.11-23.90)$ & $4.27(3.93-4.61)$ \\
\hline C32 Larynx & 11 & 16.039 & $0.69(0.34-1.24)$ & $0.46(0.23-0.82)$ & 13 & $1.57(0.78-2.81)$ & $1.72(1.58-1.85)$ & 60 & $0.78(0.39-1.40)$ & $0.78(0.72-0.85)$ \\
\hline C34 Lung & 130 & 73,984 & $1.80(1.51-2.15)$ & $1.17(0.98-1.39)$ & 258 & $0.93(0.78-1.11)$ & $1.02(0.94-1.10)$ & 559 & $1.00(0.84-1.19)$ & $0.99(0.91-1.07)$ \\
\hline C38 Heart, mediastinum and pleura & 01 & 1,488 & $0.67(0.02-3.73)$ & $0.45(0.01-2.51)$ & 03 & $0.62(0.02-3.45)$ & $0.68(0.62-0.73)$ & 07 & $0.61(0.02-3.40)$ & $0.61(0.56-0.66)$ \\
\hline C40-41 Bone and articular cartilage & 04 & 2764 & $1.45(0.39-3.71)$ & $0.97(0.26-2.48)$ & 06 & $1.24(0.34-3.17)$ & $1.35(1.24-1.46)$ & 16 & $1.07(0.29-2.74)$ & $1.07(0.98-1.15)$ \\
\hline C43-C44 Skin & 12 & 6,017 & $2.00(1.03-3.50)$ & $1.33(0.69-2.33)$ & 18 & $1.24(0.64-2.17)$ & $1.35(1.24-1.46)$ & 58 & $0.89(0.46-1.56)$ & $0.88(0.81-0.95)$ \\
\hline C43 Malignant melanoma of skin & 11 & 3,038 & $3.64(1.82-6.52)$ & $2.42(1.21-4.33)$ & 17 & $1.20(0.60-2.15)$ & $1.31(1.21-1.42)$ & 48 & $0.98(0.49-1.75)$ & $0.98(0.90-1.06)$ \\
\hline $\begin{array}{l}\text { C44 0ther malignant neoplasms of } \\
\text { skin }\end{array}$ & 01 & 2,979 & $0.34(0.01-1.89)$ & $0.22(0.01-1.22)$ & 01 & $1.86(0.05-10.36$ & $2.03(1.87-2.19$ & 10 & $0.43(0.01-2.40)$ & $0.43(0.39-0.46)$ \\
\hline C49 0ther connective and soft tissue & 03 & 1,375 & $2.19(0.45-6.39)$ & $1.46(0.30-4.26)$ & 05 & $1.11(0.22-3.24)$ & $1.22(1.12-1.32)$ & 16 & $0.80(0.16-2.34)$ & $0.80(0.74-0.86)$ \\
\hline C61 Prostate & 76 & 44,804 & $1.72(1.36-2.16)$ & $1.13(0.89-1.42)$ & 148 & $0.95(0.75-1.20)$ & $1.04(0.96-1.13)$ & 266 & $1.23(0.98-1.55)$ & $1.22(1.12-1.32)$ \\
\hline $\begin{array}{l}\text { C64-C66 Kidney. renal pélvis and } \\
\text { ureter }\end{array}$ & 10 & 5,726 & $1.75(0.84-3.22)$ & $1.17(0.56-2.15)$ & 39 & $0.47(0.23-0.86)$ & $0.52(0.48-0.56)$ & 71 & $0.60(0.29-1.10)$ & $0.60(0.55-0.65)$ \\
\hline C67 Bladder & 20 & 9,075 & $2.22(1.36-3.42)$ & $1.17(0.70-1.80)$ & 28 & $1.33(0.81-2.05)$ & $1.45(1.33-1.57)$ & 57 & $1.51(0.92-2.33)$ & $1.50(1.38-1.62)$ \\
\hline C69 Eye and adnexa & 01 & 271 & $3.70(0.09-20.6)$ & $2.47(0.06-13.6)$ & 00 & - & - & 02 & $2.14(0.05-11.92)$ & $2.13(1.96-2.30)$ \\
\hline C71Brain & 24 & 11,629 & $2.08(1.33-3.10)$ & $1.38(0.88-2.06)$ & 51 & $0.87(0.56-1.30)$ & $0.96(0.88-103)$ & 94 & $1.10(0.71-1.64)$ & $1.09(1.00-1.18)$ \\
\hline $\begin{array}{l}\text { C73-C75 Thyroid and other endocrine } \\
\text { glands }\end{array}$ & 03 & 1,590 & $1.89(0.39-5.52)$ & $1.26(0.26-3.68)$ & 07 & $0.80(0.16-2.34)$ & $0.87(0.80-0.94)$ & 14 & $0.92(0.19-2.69)$ & $0.91(0.84-0.99)$ \\
\hline C81 Hodgkin's disease & 02 & 872 & $2.30(0.28-8.30)$ & $1.53(0.18-5.52)$ & 04 & $0.93(0.11-3.36)$ & $1.01(0.93-1.10)$ & 08 & $1.07(0.13-3.86)$ & $1.07(0.98-1.15)$ \\
\hline C82-C85 Non-Hodgkin's Iymphoma & 20 & 7,937 & $2.54(1.55-3.91)$ & $1.68(1.03-2.59)$ & 58 & $0.64(0.39-0.99)$ & $0.70(0.64-0.76)$ & 106 & $0.81(0.49-1.25)$ & $0.81(0.74-0.87)$ \\
\hline C90 Multiple myeloma & 17 & 4,821 & $3.55(2.07-5.68)$ & $2.36(1.38-3.78)$ & 33 & $0.96(0.56-1.54)$ & $1.05(0.96-1.13)$ & 42 & $1.74(1.01-2.78)$ & $1.73(1.59-1.87)$ \\
\hline C91-C95 Leukemia & 29 & 8,877 & $3.30(2.21-4.75)$ & $2.18(1.46-3.14)$ & 55 & $0.98(0.66-1.41)$ & $1.07(0.98-1.16)$ & 77 & $1.62(1.09-2.33)$ & $1.61(1.48-1.74)$ \\
\hline
\end{tabular}

$\mathrm{n}$ Dent $=$ number of deaths among dentists; $n$ Phys = number of deaths among physicians; $n$ Law $=$ number of deaths among lawyers.

2.69-8.67) and a CPMR $=1.53$ (95\% C.I. 1.11-2.03) compared to the general population. The distribution of brain cancer deaths among women 20-49 yr. showed a $\mathrm{MOR}=5.56(95 \%$ C.I. 3.35-8.67) and a $\mathrm{CPMR}=2.46$ (95\% C.I. 1.48-3.84) compared to the general population; a $\mathrm{MOR}=3.33(95 \%$ C.I. $2.00-5.19)$ and a $\mathrm{CPMR}=2.66$ (95\% C.I. 2.24-3.15) compared to female physicians; and a $\mathrm{MOR}=3.42(95 \%$ C.I. 2.06-5.34) and a $\mathrm{CPMR}=2.69$ (95\% C.I. 2.27-3.19) compared to female lawyers (Table III).

Increased mortality risk estimates of thyroid cancer among female dentists were also observed compared to the general population (MOR $=10.9,95 \%$ C.I. $3.54-22.5$ and $\mathrm{CPMR}=5.00,95 \%$ C.I. $1.62-11.6$ ), compared to female physicians $(\mathrm{MOR}=1.98,95 \%$ C.I. $0.64-4.61$ and $\mathrm{CPMR}=$ $1.63,95 \%$ C.I. $1.38-1.93$ ), and compared to female lawyers $(\mathrm{MOR}=7.02,95 \%$ C.I. $2.27-16.4$ and $\mathrm{CPMR}=5.67,95 \%$ C.I. $4.78-6.71)$.

\section{Female dentists $50-79$ yr}

Compared to the general female population 50-79 yr old, females dentists in the same age group showed an all-site cancer $\mathrm{MOR}=4.22(95 \%$ C.I. $1.61-9.59)$; a $\mathrm{MOR}=1.50$ (95\% C.I. 1.27-1.77) compared to female dentists aged 50 $79 \mathrm{yr}$; and a MOR $=1.46$ (95\% C.I. 1.24-1.72) comparative to female lawyers aged 50-79 yr. When evaluating leukemia, the following cancer mortality risk estimates were ascertained: $\mathrm{MOR}=4.40 \quad(95 \%$ C.I. $1.61-9.59)$ compared to women in the general population; MOR $=3.40$, $(95 \%$ C.I. 1.25-7.41) and CPMR $=2.66$ (95\% C.I. 2.26-3.11) compared to female physicians; and MOR $=2.98$ (95\% C.I. 1.09 6.50) and $\mathrm{CPMR}=2.38(95 \%$ C.I. 2.02-2.78) compared to female lawyers (Table IV).

Increased mortality risk estimates were observed for kidney cancer among female dentists aged 50-79 yr., with a 
TABLE III. Cancer Mortality Risk, Female Dentists Comparatively to Female Physicians and Lawyers, 20-49 yr, Brazil1996-2004.

\begin{tabular}{|c|c|c|c|c|c|c|c|c|c|c|}
\hline Death causes (ICD10*) & n Dent & $\begin{array}{l}\text { Gen } \\
\text { Popul }\end{array}$ & MOR & CPMR & n Phys & $\begin{array}{c}\text { MOR } \\
\text { (CI95\%) }\end{array}$ & CPMR & n Law & $\begin{array}{c}\text { MOR } \\
\text { (Cl95\%) }\end{array}$ & CPMR \\
\hline $\begin{array}{l}\text { C00-C97 All Malignant } \\
\text { neoplasms }\end{array}$ & 142 & 97,745 & $2.26(1.91-2.67)$ & & 139 & $1.35(1.14-1.60)$ & & 322 & $1.38(1.17-1.63)$ & \\
\hline C16 Stomach & 05 & 5,110 & $1.46(0.47-3.40)$ & $0.67(0.22-1.56)$ & 07 & $0.84(0.27-1.96)$ & $0.70(0.59-0.83)$ & 10 & $1.39(0.45-3.24)$ & $1.13(0.96-1.34)$ \\
\hline C18-21 Colon-rectum & 09 & 5,496 & 2.47 (1.13-4.69) & $1.28(0.59-2.43)$ & 12 & $0.88(0.40-1.67)$ & $0.73(0.62-0.87)$ & 26 & $0.96(0.44-1.82)$ & $0.78(0.66-0.93)$ \\
\hline $\begin{array}{l}\text { C22 Liver and intra-hepatic } \\
\text { bile ducts }\end{array}$ & 04 & 2,659 & $2.25(0.61-5.76)$ & $1.03(0.35-3.28)$ & 03 & $1.58(0.43-4.04)$ & $1.31(1.10-1.55)$ & 10 & $1.11(0.30-2.84)$ & $0.91(0.77-1.07)$ \\
\hline $\begin{array}{l}\text { C23-C24 Gallbladder and } \\
\text { other unspecified parts } \\
\text { of biliary tract }\end{array}$ & 01 & 1,093 & $1.36(0.03-7.58)$ & $0.63(0.01-3.51)$ & 01 & $1.18(0.03-6.57)$ & $0.98(0.83-1.16)$ & 01 & $2.78(0.07-15.48)$ & $2.27(1.91-2.68)$ \\
\hline C25 Pancreas & 01 & 1,578 & $0.94(0.02-5.24)$ & $0.44(0.01-2.45)$ & 04 & $0.29(0.01-1.62)$ & $0.24(0.20-0.29)$ & 12 & $0.23(0.01-1.28)$ & $0.19(0.16-0.22)$ \\
\hline $\begin{array}{l}\text { C30-31 Nasal cavity. } \\
\text { middle ear and } \\
\text { accessory sinuses }\end{array}$ & 01 & 117 & $12.75(0.32-71.0)$ & $5.88(0.15-32.8)$ & 00 & - & - & 00 & - & - \\
\hline C34 Bronchus and lung & 08 & 5,397 & $2.23(0.96-4.39)$ & $1.02(0.44-2.01)$ & 13 & $0.72(0.31-1.42)$ & $0.60(0.51-0.71)$ & 30 & $0.73(0.31-1.44)$ & $0.60(0.51-0.72)$ \\
\hline $\begin{array}{l}\text { C40-41 Bone and articular } \\
\text { cartilage }\end{array}$ & 01 & 1,011 & $1.47(0.04-8.19)$ & $0.68(0.08-3.79)$ & 02 & $0.59(0.01-3.29)$ & $0.49(0.41-0.58)$ & 02 & $1.39(0.04-7.74)$ & $1.13(0.96-1.34)$ \\
\hline C43-C44 Skin & 03 & 1,499 & $2.99(0.62-8.73)$ & $1.38(0.28-4.03)$ & 02 & $1.78(0.37-5.20)$ & $1.47(1.24-1.74)$ & 05 & $1.67(0.34-4.88)$ & $1.36(1.15-1.61)$ \\
\hline $\begin{array}{l}\text { C43 Malignant } \\
\text { melanoma of skin }\end{array}$ & 03 & 1,141 & $3.94(0.81-11.50)$ & $1.81(0.37-\mathbf{X}$ & 02 & $1.78(0.37-5.20)$ & $1.47(1.24-1.74)$ & 05 & $1.67(0.34-4.88)$ & $1.36(1.15-1.61)$ \\
\hline $\begin{array}{l}\text { C44 0ther malignant } \\
\text { neoplasms of skin }\end{array}$ & 00 & - & - & - & 00 & - & - & 00 & - & - \\
\hline $\begin{array}{l}\text { C49 0ther connective and } \\
\text { soft tissue }\end{array}$ & 01 & 358 & 0 & 0 & 02 & $0.59(0.01-3.29)$ & $0.49(0.41-0.58)$ & 02 & $1.39(0.04-7.74)$ & $1.13(0.96-1.34)$ \\
\hline C50 Breast & 47 & 21,152 & $3.67(2.69-4.89)$ & $1.53(1.11-2.03)$ & 41 & $1.41(1.04-1.88)$ & $1.12(0.95-1.33)$ & 102 & $1.32(0.97-1.76)$ & $1.04(0.88-1.24)$ \\
\hline C53-C55 Uteri & 08 & 1,853 & $0.63(0.27-1.24)$ & $0.30(0.13-0.59)$ & 03 & $3.20(1.38-6.30)$ & $2.61(2.20-3.09)$ & 23 & $0.96(0.41-1.89)$ & $0.79(0.67-0.93)$ \\
\hline C53 Cervix uteri & 04 & 13,210 & $0.44(0.12-1.33)$ & $0.21(0.06-2.54)$ & 00 & - & - & 16 & $0.69(0.19-1.77)$ & $0.57(0.48-0.67)$ \\
\hline C54 Corpus uteri & 01 & 508 & $2.93(0.07-16.32)$ & $1.36(0.03-7.57)$ & 02 & $0.59(0.01-3.29)$ & $0.49(0.41-0.58)$ & 01 & $2.78(0.07-15.48)$ & $2.27(1.91-2.68)$ \\
\hline $\begin{array}{l}\text { C55 Uterus. part } \\
\text { unspecified }\end{array}$ & 03 & $x x x x x x$ & $0.93(0.19-2.72)$ & $0.47(0.01-1.26)$ & 01 & $3.57(0.74-10.42)$ & $2.94(2.48-3.48)$ & 06 & $1.39(0.29-4.06)$ & $1.13(0.96-1.34)$ \\
\hline C56 Ovary & 04 & 4,815 & $1.53(0.42-3.92)$ & $0.71(0.19-1.82)$ & 09 & $0.52(0.14-1.33)$ & $0.44(0.37-0.52)$ & 13 & $0.85(0.23-2.18)$ & $0.70(0.59-0.83)$ \\
\hline C58 Placenta & 01 & 121 & $12.3(0.31-68.6)$ & $5.69(0.14-31.7)$ & 01 & $1.18(0.03-6.57)$ & $0.98(0.83-1.16)$ & 00 & - & - \\
\hline C71Brain & 19 & 5,324 & $5.56(3.35-8.67)$ & $2.46(1.48-3.84)$ & 07 & $3.33(2.00-5.19)$ & $2.66(2.24-3.15)$ & 16 & $3.42(2.06-5.34)$ & $2.69(2.27-3.19)$ \\
\hline $\begin{array}{l}\text { C73-C75 Thyroid and other } \\
\text { endocrine glands }\end{array}$ & 05 & 689 & $10.9(3.54-25.5)$ & $5.00(1.62-11.6)$ & 03 & $1.98(0.64-4.61)$ & $1.63(1.38-1.93)$ & 02 & $7.02(2.27-16.36)$ & $5.67(4.78-6.71)$ \\
\hline C81 Hodgkin's disease & 01 & 660 & $2.24(0.06-12.6)$ & $1.04(0.03-5.79)$ & 03 & $0.39(0.01-2.17)$ & $0.33(0.28-0.39)$ & 03 & $0.92(0.02-5.12)$ & $0.76(0.64-0.89)$ \\
\hline $\begin{array}{l}\text { C82-C85 Non-Hodgkin's } \\
\text { lymphoma }\end{array}$ & 05 & 2,360 & $3.18(1.03-7.41)$ & $1.46(0.47-3.40)$ & 03 & $1.98(0.64-4.61)$ & $1.63(1.38-1.93)$ & 07 & $1.99(0.64-4.64)$ & $1.62(1.37-1.92)$ \\
\hline C91-C95 Leukemia & 06 & 5,045 & $1.78(0.65-3.88)$ & $6.82(0.30-1.79)$ & 06 & $1.18(0.43-2.57)$ & $0.98(0.83-1.16)$ & 20 & $0.83(0.30-1.81)$ & $0.68(0.57-0.81)$ \\
\hline
\end{tabular}

$\mathrm{n}$ Dent $=$ number of deaths among dentists; $\mathrm{n}$ Phys $=$ number of deaths among physicians; $\mathrm{n}$ Law $=$ number of deaths among lawyers.

$\mathrm{MOR}=4.80$ (95\% C.I. 0.99-14.0) compared to females in the general population, a MOR $=5.07(95 \%$ C.I. $1.04-14.8)$ compared to female physicians, and a $\mathrm{MOR}=3.99$ $(95 \%$ C.I. $=3.39-4.67)$ compared to female lawyers (Table IV).

\section{DISCUSSION}

In Brazil, as in many other developing countries, regulatory measures concerning occupational and environmental health are usually considered to be inadequate. In addition, insufficient surveillance, which is often based on outdated environmental threshold limit values and biological exposure indices, may amplify the exposure of Brazilian professionals, such as dentists, to certain risk factors, including potentially carcinogenic risk factors present in the occupational environment. Accordingly, we hypothesized that Brazilian dentists may be at a higher risk of death for specific types of cancer. To test this hypothesis, the present study used records of death certificates in a National Mortality System to compare the cancer mortality profile among Brazilian dentists with that experienced during 19962004 by the general population and selected occupational categories (physicians and lawyers).

In fact, our results suggest that Brazilian dentists are more likely to die from cancer at certain sites compared to physicians, lawyers, and the general population. In general, 
TABLE IV. Cancer Mortality Risk, Female Dentists Comparatively to Female Physicians and Lawyers, 50-79yr, Brazil, $1996-2004$.

\begin{tabular}{|c|c|c|c|c|c|c|c|c|c|c|}
\hline \multirow[b]{2}{*}{ Death causes (ICD10*) } & \multicolumn{3}{|c|}{ N Gen } & \multirow[b]{2}{*}{ CPMR (95\%CI) } & \multirow[b]{2}{*}{ n Phys } & \multirow[b]{2}{*}{ MOR (95\% CI) } & \multirow[b]{2}{*}{ CPMR (95\% CI) } & \multirow[b]{2}{*}{ n Law } & \multirow[b]{2}{*}{ MOR (95\% CI) } & \multirow[b]{2}{*}{ CPMR (95\% CI) } \\
\hline & n Dent & Pop & MOR (95\% CI) & & & & & & & \\
\hline C00-C97 All Malignant neoplasms & 148 & 309,776 & $4.22(3.58-4.97)$ & & 197 & $1.50(1.27-1.77)$ & & 645 & $1.46(1.24-1.72)$ & \\
\hline C00-C14 Oral Cavity & 01 & 5,001 & $1.12(0.02-6.24)$ & $0.42(0.01-2.34)$ & 02 & $0.84(0.02-4.68)$ & $0.67(0.57-0.78)$ & 07 & $0.77(0.02-4.29)$ & $0.62(0.53-0.73)$ \\
\hline C16 Stomach & 05 & 21,699 & $1.29(0.42-3.01)$ & $0.48(0.16-1.12)$ & 06 & $1.40(0.45-3.26)$ & $1.11(0.94-1.30)$ & 20 & $1.35(0.43-3.15)$ & $1.09(0.93-1.27)$ \\
\hline C17 Small intestine & 01 & 920 & $6.08(0.15-33.9)$ & & 00 & - & - & 02 & $2.70(0.07-15.04)$ & $2.18(1.85-2.55)$ \\
\hline C18-21Colorectal & 13 & 23,598 & $3.12(1.66-5.34)$ & $2.27(1.21-3.88)$ & 20 & $1.09(0.58-1.86)$ & $0.87(0.74-1.01)$ & 62 & $1.13(0.60-1.93)$ & $0.91(0.78-1.07)$ \\
\hline C22 Liver & 02 & 13,922 & $0.80(0.10-2.89)$ & $0.30(0.04-1.08)$ & 10 & $0.33(0.04-1.19)$ & $0.27(0.23-0.31)$ & 24 & $0.45(0.05-1.62)$ & $0.39(0.31-0.42)$ \\
\hline $\begin{array}{l}\text { C23-C24 Gallbladder and other } \\
\text { unspecified parts of biliary tract }\end{array}$ & 02 & 9,767 & $1.14(0.14-4.12)$ & $0.43(0.01-1.55)$ & 01 & $3.37(0.41-12.17)$ & $2.66(2.26-3.11)$ & 10 & $1.08(0.13-3.90)$ & $0.87(0.74-1.02)$ \\
\hline C25 Pancreas & 06 & 13,831 & $2.45(0.90-5.34)$ & $0.91(0.33-1.98)$ & 12 & $0.84(0.31-1.83)$ & $0.67(0.57-0.78)$ & 42 & $0.76(0.28-1.66)$ & $0.62(0.53-0.73)$ \\
\hline C32 Larynx & 01 & 2,040 & $2.74(0.07-15.3)$ & $1.03(0.03-5.73)$ & 00 & - & - & 01 & $5.40(0.14-30.08)$ & $4.36(3.70-5.10)$ \\
\hline C34 Lung & 11 & 29,810 & $2.10(1.05-3.76)$ & $0.78(0.39-1.40)$ & 25 & $0.73(0.36-1.31)$ & $0.59(0.50-0.69)$ & 90 & $0.65(0.32-1.16)$ & $0.53(0.45-0.62)$ \\
\hline C43-C44 Skin & 06 & 3,628 & $9.39(3.45-20.5)$ & $3.46(1.27-7.54)$ & 00 & - & - & 04 & $8.22(3.02-17.92)$ & $6.54(5.56-7.65)$ \\
\hline C43 Malignant melanoma of skin & 04 & 2,098 & $10.8(2.93-27.6)$ & $3.99(1.08-10.2)$ & 00 & - & - & 03 & $7.26(1.97-18.59)$ & $5.81(4.94-6.80)$ \\
\hline C44 Other malignant neoplasms of skin & 02 & 1,530 & $7.33(0.89-26.5)$ & $2.74(0.33-9.89)$ & 00 & - & - & 01 & $10.84(1.31-39.13)$ & $8.72(7.41-10.20)$ \\
\hline C50 Breast & 39 & 46,682 & $5.19(3.69-7.09)$ & $1.75(1.04-2.41)$ & 52 & $1.29(0.92-1.76)$ & $1.00(0.85-1.17)$ & 171 & $1.26(0.90-1.72)$ & $0.99(0.84-1.16)$ \\
\hline C53-C55 Uteri & 14 & 35,726 & $2.24(1.22-3.76)$ & $0.82(0.48-1.38)$ & 12 & $2.00(1.09-3.36)$ & $1.55(1.32-1.82)$ & 30 & $2.59(1.41-4.35)$ & $2.03(1.73-2.38)$ \\
\hline C53 Cervix uteri & 06 & 19,490 & $1.73(0.63-3.77)$ & $0.64(0.23-1.39)$ & 03 & $3.40(1.25-7.41)$ & $2.66(2.26-3.11)$ & 04 & $8.22(3.02-17.92)$ & $6.54(5.56-7.65)$ \\
\hline C54 Corpus uteri & 03 & 4,428 & $3.81(0.78-11.1)$ & $1.42(0.29-4.15)$ & 04 & $1.26(0.26-3.68)$ & $1.00(0.85-1.17)$ & 14 & $1.16(0.24-3.39)$ & $0.93(0.79-1.09)$ \\
\hline C55 Uterus. part unspecified & 05 & 11,808 & $2.38(0.77-5.55)$ & $0.89(0.29-2.07)$ & 05 & $1.69(0.55-3.94$ & $1.33(1.13-1.56)$ & 12 & $2.26(0.73-5.27)$ & $1.82(1.54-2.12)$ \\
\hline C56 Ovary & 09 & 11,902 & $4.32(1.97-2.81)$ & $1.58(0.72-3.00)$ & 10 & $1.52(0.70-2.89)$ & $1.20(1.02-1.40)$ & 49 & $0.99(0.45-1.88)$ & $0.80(0.68-0.94)$ \\
\hline C64-C66 Kidney. renal pélvis and ureter & 03 & 3,516 & $4.80(0.99-14.0)$ & $1.79(0.37-5.23)$ & 01 & $5.07(1.04-14.80)$ & $3.99(3.39-4.67)$ & 05 & $3.25(0.67-9.49)$ & $2.61(2.22-3.06)$ \\
\hline C71Brain & 05 & 10,315 & $2.73(0.88-6.36)$ & $1.01(0.33-2.35)$ & 04 & $2.11(0.68-4.92)$ & $1.66(1.41-1.95)$ & 22 & $1.23(0.40-2.87)$ & $0.99(0.84-1.16)$ \\
\hline C82-C85 Non-Hodgkin's lymphoma & 07 & 6,450 & $6.17(2.47-12.7)$ & $2.27(0.91-4.68)$ & 10 & $1.18(0.47-2.43)$ & $0.93(0.79-1.09)$ & 18 & $2.12(0.85-4.37)$ & $1.69(1.44-1.98)$ \\
\hline C90 Multiple myeloma & 01 & 4,779 & $1.17(0.02-6.52)$ & $0.44(0.01-2.44)$ & 04 & $0.42(0.01-2.34)$ & $0.33(0.28-0.39)$ & 12 & $0.45(0.01-2.51)$ & $0.36(0.31-0.42)$ \\
\hline C91-C95 Leukemia & 06 & 7,727 & $4.40(1.61-9.59)$ & $1.62(0.59-3.53)$ & 03 & $3.40(1.25-7.41)$ & $2.66(2.26-3.11)$ & 11 & $2.98(1.09-6.50)$ & $2.38(2.02-2.78)$ \\
\hline
\end{tabular}

$\mathrm{n}$ Dent $=$ number of deaths among dentists; $\mathrm{n}$ Phys = number of deaths among physicians; $\mathrm{n}$ Law $=$ number of deaths among lawyers.

larger MORs was observed in dentists compared to the general population. However, the comparisons performed with physicians and lawyers also revealed some impressively high mortality risk estimates in dentists. It is noteworthy that the risk of death as a consequence of leukemia, brain, and breast neoplasms among female dentists was several times higher than that expected based on the cause-specific mortality rates of the chosen reference groups.

These results could be affected by several factors. At first, cancer deaths may be under-reported in the general population, considering that a percentage of patients in the terminal stage of cancer can die in their homes without proper diagnosis or attribution. Hence, cancer mortality comparisons performed between dentists and the general population may be somehow overestimated.

The current results could also be affected by confounding introduced by different income distributions in the reference populations. Indeed, the monthly mean income among Brazilian physicians was twice as high as that verified among Brazilian dentists and lawyers. Nevertheless, it was considered justifiable to include the former as a comparison group because both physicians and dentists work in health field activities and experience similar exposure conditions, such as biological, physical, and chemical agents. However, the consistently higher mortality risk estimates for some types of cancer among dentists compared to all three reference groups seems to be evidence that dentists may in fact be at a higher risk of developing such neoplasms.

Many of the comparisons conducted in this study included a very small number of deaths, and therefore, the related risk estimates should be analyzed cautiously. Considering that, such analyses are likely to be unstable, only results with statistically significant or borderline risk estimates were discussed in the text.

Higher risks of all cancer mortality rates compared to the general population were found for both younger (20-49 yr.) and older (50-79 yr.) male and female dentists. Additionally, 
statistically significant higher risks of soft tissue sarcoma were observed for male dentist ages 20-49 yr., compared to male physicians and male lawyers.

Female dentists had higher all-cancer mortality risks than the general population, female dentists and female lawyers at both age ranges (20-49 yr. and 50-79 yr.) Higher risk estimates for female dentists, reported by MORs and CPMRs using several comparison groups were observed for brain cancer at ages 20-49 yr. and leukemia at ages 50-79 yr.

Among female dentists ages 20-49 yr, higher brain cancer mortality risk estimates were observed compared to the general population, to female physicians and female lawyers (Table III). When evaluating thyroid cancer, female dentists showed higher mortality risk estimates compared to female lawyers (Table III). It is important to note that these high-risk estimates of thyroid cancer may result from continuous occupational exposure to ionizing radiation, whose causal association with such neoplasia is well established in the literature [Memon et al., 2010].

Comparisons performed in the 50-79 yr. age group revealed that female dentists showed higher risk estimates for leukemia compared to the general population, to female physicians and to female lawyers (Table IV).

Higher risks estimates were also observed for kidney cancer among female dentists compared to the general population and to female physicians (Table IV). In contrast, the determined CPMRs of kidney cancer compared to the general population and to physicians were not statistically significant.

When evaluating skin melanoma in women aged 5079 yr., higher risk estimates were observed among female dentists compared to the general population and to female lawyers.

Among male dentists ages $20-49$ yr., higher mortality risk estimates were observed for bone and articular cartilage tumors compared to lawyers (Table I). Among males ages 50-79 yr., increased statistically significant MORs and CPMRs compared to the general population, but not relative to physicians or lawyers, were observed for pancreas, lung, non-Hodgkin lymphoma, multiple myeloma, and leukemia. In the same age group, increased risk estimates were also observed for prostate cancer among dentists compared to lawyers (Table II). Compared to male lawyers, a higher risk of leukemia mortality among male dentists was also observed.

Dentists' occupational activity encompasses environmental exposures to potentially carcinogenic agents originating from several sources, including biological (following close contact with human tissues and saliva), physical (such as ionizing radiation) and a large array of chemical substances, including solvents and metals [Szymanska 1999; Al-Khatib et al., 2006].

In fact, some of the most commonly used materials and processes in dental practice have already been evaluated and classified according to their carcinogenic potential to humans by the International Agency for Research on Cancer (IARCWHO). Hence, chromium ( $\mathrm{Cr}$ ) and beryllium $(\mathrm{Be})$, which are used in metal alloys for dental restorations [Wang and $\mathrm{Li}, 1998$ ] and in dental prosthesis [Gordon and Bowser, 2003], respectively, have been categorized as carcinogenic to humans and therefore are included in Group 1 of the IARC classification of human carcinogens [Gonzalez and Agudo, 1999; Beyersmann, 2002; Siemiatycki et al., 2004; Clapp et al., 2007]. Similarly, the current use of asbestos in dental materials has also been highlighted to be an important risk factor for the development of lung cancer among dentists [Fry, 2009]. Ionizing radiation, to which dentists are exposed when performing diagnostic X-rays, is also carcinogenic [Brenner et al., 2003; Siemiatycki et al., 2004; Zielinski et al., 2005; Clapp et al., 2007]. In addition, formaldehyde, an antimicrobial drug used for intra-channel dressings in endodontics [Lewis and Chestner, 1981], has also been included in IARC Group $1 \mathrm{~A}$ as being carcinogenic to humans (IARC \WHO, 2013). Other chemicals and metals to which dentists experience long-term exposure, with debatable carcinogenic effects, include nitrous oxide, which is used in short-term anesthesia [Szymanska, 2001], and mercury, which is used to prepare amalgam to fill dental cavities [Perez-Gomez et al., 2005].

In this investigation, stratification by gender and age seems to have uncovered differences in the outcomes or risk levels among dentists. Even when professionals of both genders perform identical job tasks, with similar external exposures, the absorbed dose may be higher among men as a consequence of their larger surface area, which provides more opportunity for dermal absorption. Larger lung volumes among men could also have a similar effect on inhalation exposure. However, women have thinner skin in many areas of the body, a smaller volume of total body water, and a higher percentage of body fat, which can allow easier penetration, absorption, and storage of chemicals [Silvaggio and Mattison, 1992]. According to some authors, the pattern of risk could also be associated with duration of work, age, calendar time of initial employment, timing, level, and period of exposures [Wang et al., 1990, Blair et al., 1999]. Altogether, this scenario of continuous exposure to several risk factors for decades of activity in the dentistry practice may create conditions that are favorable for carcinogenesis [Simning and van Wijngaarden, 2007].

Studies using mortality databases, such as ours, are empirical investigations involving the analysis of data available on death certificates in large populations. The cost-efficiency advantage offered by this type of epidemiologic design makes it suitable for exploring scientific hypotheses that can be further tested using more robust studies. However, it is also true that results obtained by such designs should be considered in light of several limitations [Redelings et al., 2007]. First, death certificate data are generally analyzed by examining the underlying cause of 
death as reported by the medical examiner. However, deaths rarely have only one unique cause, and this method does not allow researchers to assess the role of adjacent conditions that may have been important contributors to death [Redelings et al., 2007]. Furthermore, because death registrations only record the usual occupation at the time of death, changes in the type or duration of a job over time cannot be assessed [MacArthur et al., 2007]. In addition, inconsistencies regarding occupational histories may also occur when a person has held more than one job. Schade and Swanson (1988) reported a $40 \%$ error rate of "usual occupation" recorded on death certificates when compared with occupational histories. However, dentists usually have their occupation for life in Brazil, and 'multiple occupations' as a potential for misclassification does not seem to play a role in this study.

Mortality estimates obtained from death certificates can also be a poor risk indicator for several types of cancer. For instance, the high survival rates observed for some types of cancer, such as thyroid and non-melanoma skin cancers, indicate that mortality studies could be missing some incident cases, thereby abating the magnitude of the association observed for some occupations [MacArthur et al., 2007]. Moreover, one of the major limitations of this study is that multiple comparisons were made. It is therefore possible that some associations were due to chance, especially for those cancers with small numbers.

In the present study, causal inference was also limited due to the lack of precise individual information on exposure levels to occupational risk factors, as well as to potential confounding factors, such as smoking status, alcohol consumption and dietary intake. However, according to $\mathrm{Ji}$ and Hemminki (2005), Swedish dentists are commonly nonsmokers and most likely do not consume alcohol excessively. In Brazil, however, a study performed in Brasilia in 2004 with 498 dentists reported that $37 \%$ of dentists ( $42 \%$ of males and $32 \%$ of females) were reported to be smokers (Rodrigues et al., 2008). In another study performed with a populationbased sample of 100 dentists in Porto Alegre, Rio Grande do Sul state, a smoking prevalence of $14.3 \%$ among male dentists and 5.9\% among female dentists was observed. Additionally, the respective prevalence rates of alcohol intake estimates were $71.4 \%$ and $35.3 \%$ among males and females [Lopes and Amaral, 2005].

According to the other occupational groups analyzed in this study, a survey performed with 5,158 physicians in 23 Brazilian states in the late 1990's reported a smoking prevalence of $24.9 \%$, with smoking rates reported to be higher among males than females [Campos, 1992]. In a crosssectional study performed with fourth year medical students in the state of Minas Gerais, Brazil, $60 \%$ of the participants reported the consumption of alcoholic beverages in the last month before interview. Additionally, $25 \%$ of these students evidenced the need for educational programs and interventions to prevent the use of alcoholic beverages [Rocha et al., 2011]. Similar reports about the prevalence of smoking and alcohol intake among lawyers in Brazil could not be identified in the literature.

One final possible limitation in our study is the healthy worker effect. An individual must be relatively healthy in order to be employable in the workforce, and therefore, both morbidity and mortality rates within the workforce are usually lower than those occurring in the general population. As a result, a true excess in both disease incidence and mortality estimates due to harmful exposures at work might be wholly or partially diminished [Li and Sung, 1999]. In the present study, cancer mortality profiles between Brazilian dentists and other population groups, including the general population, were compared. Thus, the occurrence of the healthy worker effect cannot be ruled out. Nevertheless, the inclusion of physician and lawyer mortality data in the analysis attempted to reduce any misinterpretations resulting from a healthy worker effect introduced in the performed data analysis with the general population. Additionally, many investigators have argued that the healthy worker effect is of little or no consequence in interpreting data on cancer mortality. It is unlikely that factors predicting eventual cancer deaths would be present at 20 yr of age when many people become employed [Li and Sung, 1999]. Moreover, if the healthy worker effect did occur, it would artificially reduce the ascertained risk estimates among the studied group (dentists) in this investigation, and not the inverse. In our study, sensitivity analysis performed after excluding controls with cardiovascular diseases or external causes of death did not substantially modify the previously estimated cancer mortality risks among dentists.

\section{CONCLUSIONS}

The results from this study revealed that mortality for several types of cancer was significantly elevated among Brazilian dentists 20-79 yr old compared with other selected groups during 1996 to 2004 . The most commonly diagnosed tumors include soft tissue, brain, breast, thyroid, melanoma, kidney, multiple myeloma, and leukemia. Stratified analyses showed specific patterns of cancer mortality risk according to sex and age strata. These results indicate the need for limiting occupational exposures among dentists, mainly to specific chemicals. The results also suggest a need to establish screening programs with the aim of achieving early detection of selected malignant tumors, particularly among female dentists.

\section{ACKNOWLEDGEMENTS}

The current study was sponsored by different research grants from the Brazilian National Research Council-CNPq and the State of Rio de Janeiro Research Foundation 
(FAPERJ). It was also supported by a Public Health Residency scholarship from the Ministry of Health of Brazil, and a fellowship granted by the Brazilian Ministry of Education - Post-graduation Board (CAPES).

\section{REFERENCES}

Ahlbom A, Norell S, Rodvall Y, Nylander M. 1986. Dentists, dental nurses, and brain tumours. Br. Med. J 292:662.

Al-Khatib IA, Ishtayeh M, Barghouty H, Akkawi B. 2006. Dentists' perceptions of occupational hazards and preventive measures in East Jerusalem. East Mediterr. Health J 12:153-160.

Antunes Jl, Macedo MM, de Araujo ME. 2004. [Comparative analysis of cause-specific mortality for dentists in the city of Sao Paulo]. Cad. Saude. Publica 20:241-248.

Beyersmann D. 2002. Effects of carcinogenic metals on gene expression. Toxicol. Lett 127:63-68.

Blair A, Walrath J, Rogot E. 1985. Mortality patterns among U.S. veterans by occupation. I. Cancer. J. Natl. Cancer Inst 75:1039-1047.

Blair A, Zahm SH, Silverman DT. 1999. Occupational cancer among women: Research status and methodologic considerations. Am. J. Ind. Med 36:6-17.

Brenner DJ, Doll R, Goodhead DT, Hall EJ, Land CE, Little JB, Lubin JH, Preston DL, Preston RJ, Puskin JS. et al. 2003. Cancer risks attributable to low doses of ionizing radiation: Assessing what we really know. Proc. Natl. Acad. Sci. USA 100:13761-13766.

Campos HS. 1992. Smoking habits among Brazilian physicians. J Pneumol 18:1-9.

Carozza SE, Wrensch M, Miike R, Newman B, Olshan AF, Savitz DA, Yost M, Lee M. 2000. Occupation and adult gliomas. Am. J. Epidemiol 152:838-846.

Clapp RW, Howe GK, Jacobs MM. 2007. Environmental and occupational causes of cancer: A call to act on what we know. Biomed. Pharmacother 61:631-639.

Eriksson M, Hardell L, Malker H, Weiner J. 1998. Increased cancer incidence in physicians, dentists, and health care workers. Oncol. Rep 5:1413-1418.

Fry C. 2009. An investigation with asbestos related disease in the dental industry. Br. Dent. J 206:515-516.

Gonzalez CA, Agudo A. 1999. Occupational cancer in Spain. Environ. Health Perspect 107:273-277.

Gordon T, Bowser D. 2003. Beryllium: genotoxicity and carcinogenicity. Mutat. Res 533:99-105.

Hostettler M, Minder C. 2002. Mortality of Swiss dentists. Schweiz. Monatsschr. Zahnmed 112:456-462.

IARC $\backslash$ WHO. 2013. IARC monographs on the evaluation of carcinogenic risk to humans. Monographs.iarc.fr $\backslash E N G \backslash$ classification.

Ji J, Hemminki K. 2005. Occupation and upper aerodigestive tract cancers: A follow-up study in Sweden. J. Occup. Environ. Med 47:785795 .

Jorge Me, Gotlieb SLD, Laurenti R. 2002. The national mortality information system: Problems and proposals for solving them I - Deaths by natural causes. Rev Bras Epidemiol 5:197-211.

Krishnan G, Felini M, Carozza SE, Miike R, Chew T, Wrensch M. 2003. Occupation and adult gliomas in the San Francisco bay area. J Occup. Environ. Med 45:639-647.
Krstev S, Baris D, Stewart PA, Hayes RH, Blair A, Dosemeci M. 1998. Risk for prostate cancer by occupation and industry: A 24-state death certificate study. Am. J. Ind. Med 34:413-420.

Lewis BB, Chestner SB. 1981. Formaldehyde in dentistry: A review of mutagenic and carcinogenic potential. J. Am. Dent. Assoc 103:429434.

Li CY, Sung FC. 1999. A review of the healthy worker effect in occupational epidemiology. Occup. Med 49:225-229.

Linet MS, Malker HS, Chow WH, McLaughlin JK, Weiner JA, Stone BJ, Ericsson JL, Fraumeni JF Jr. 1995. Occupational risks for cutaneous melanoma among men in Sweden. J. Occup. Environ. Med 37:11271135 .

Lopes R, Amaral FG. Risk factors associated to dentists health. 2005. XXV Encontro Nacional de Engenharia de Produção, Porto Alegre. www.abepro.og.br/biblioteca/ENEGEP 2005_Enegep04008_1612.pdf

MacArthur AC, Le ND, Abanto ZU, Gallagher RP. 2007. Occupational female breast and reproductive cancer mortality in British Columbia, Canada,1950-94. Occup. Med 57:246-253.

Memon A, Godward S, Williams D, Siddique I, Al-Saleh K. 2010. Dental x-rays and the risk of thyroid cancer: A case-control study. Acta Oncol 49(4):447-453.

Miettinen OS, Wang J.-D. 1981. An alternative to the proportionate mortality ratio. Am J Epidemiol 114:144-148.

Monteiro GT, Koifman RJ, Koifman S. 1997. Reliability and accuracy of reported causes of death from cancer. II accuracy of stomach cancer reported in the municipality of Rio de Janeiro County, Brazil. Cad Saude Publica 13(Suppl 1):53-65.

Meyer A, Chrisman JR, Moreira JC, Koifman S. 2003. Cancer mortality among agricultural workers from Serrana Region, state of Rio de Janeiro, Brazil. Environ. Res 93:264-271.

Morton W, Marjanovic D. 1984. Leukemia incidence by occupation in the Portland-Vancouver Metropolitan area. Am. J. Ind. Med 6:185-205.

Navas-Acien A, Pollan M, Gustavsson P, Plato N. 2002. Occupation, exposure to chemicals and risk of gliomas and meningiomas in Sweden. Am. J. Ind. Med 42:214-227.

Nishio N, Tanaka H, Tsukuma H, Tokunaga R. 2004. Lung cancer risk in male dentists: A retrospective cohort study in Japan, 1964-1997. J. Occup. Health 46:37-42.

Perez-Gomez P, Aragones N, Gustavsson P, Plato N, Lopez-Abente G, Pollan M. 2005. Cutaneous melanoma in Swedish women: occupational risks by anatomic site. Am. J. Ind. Med 48:270-281.

Perez-Gomez P, Pollan M, Gustavsson P, Plato N, Aragones N, Lopez-Abente G. 2004. Cutaneous melanoma: hints from occupational risks by anatomic site in Swedish men. Occup. Environ. Med 61:117126.

Pollan M, Gustavsson P. 1999. High-risk occupations for breast cancer in the Swedish female working population. Am. J. Public Health 89:875-881.

Preston-Martin S. 1989. Descriptive epidemiology of primary tumors of the brain, cranial nerves and cranial meninges in Los Angeles County. Neuroepidemiology 8:283-295.

Redelings MD, Wise M, Sorvillo F. 2007. Using multiple cause-of-death data to investigate associations and causality between conditions listed on the death certificate. Am. J. Epidemiol 166:104-108.

Rodrigues GA, Galvão V, Viegas CA. 2008. Prevalence of smoking among dentists in the federal district of Brasília, Brazil. J Bras Pneumol 34(5):288-293.

Rix BA, Lynge E. 1996. Cancer incidence in Danish health care workers. Scand. J. Soc. Med 24:114-120. 
Rix BA, Torsten S, Lynge E. 1997. Socioeconomic group, occupation and incidence of breast cancer and genital cancer among women in Denmark. Eur. J. Public. Health 7:177-181.

Rocha LA, Lopes ACFMM, Martelli DRB, Lima VB, Martelli H Jr. 2011. Alcohol use by medical students in Minas Gerais State. Rev Bras Educação Medica 35(3):369-375.

Sankila R, Karjalainen S, Laara E, Pukkala E, Teppo L. 1990. Cancer risk among health care personnel in Finland, 1971-1980. Scand. J. Work. Environ. Health 16:252-257.

Schade WJ, Swanson GM. 1988. Comparison of death certificate occupation and industry data with lifetime occupational histories obtained by interview: Variations in the accuracy of death certificate entries. Am. J. Ind. Med 14:121-136.

Siemiatycki J, Richardson L, Straif K, Latreille B, Lakhani R, Campbell S, Rousseau MC, Boffetta P. 2004. Listing occupational carcinogens. Environ. Health. Perspect 112:1447-1459.

Silvaggio T, Mattison DR. 1992. Setting occupational health standards: Toxicokinetic differences among and between men and women. J. Occup. Med 36:849-854.

Simning A, van Wijngaarden E. 2007. Literature review of cancer mortality and incidence among dentists. Occup. Environ. Med 64:432438.

Stewart W, Hunting K. 1988. Mortality odds ratio, proportionate mortality ratio, and healthy worker effect. Am J Ind Med 14:345353.

Szymanska J. 2001. Environmental health risk of chronic exposure to nitrous oxide in dental practice. Ann. Agric. Environ. Med 8:119122.

Szymanska J. 1999. Occupational hazards of dentistry. Ann. Agric. Environ. Health 6:13-19.

Tanaka H, Nishio N, Tokunaga R, Tsukuma H. 2004. Liver cancer in Japanese male dentists: A long term retrospective cohort study. J. Occup. Health 46:398-402.
Vagero D, Swerdlow AJ, Beral V. 1990. Occupation and malignant melanoma: A study based on cancer registration data in England and Wales and in Sweden. Br. J. Ind. Med 47:317-324.

Wang JD, Miettinen OS. 1984. The mortality odds ratio (MOR) in occupational mortality studies - selection of reference occupation(s) and reference cause(s) of death. Ann. Acad. Med. Singapore 13(Suppl 2):312-316.

Wang JX, Inskip PD, Boice JD, Li BX, Zhang JY, Fraumeni JF Jr. 1990. Cancer incidence among medical diagnostic $\mathrm{X}$-ray workers in China, 1950-1985. Int. J. Cancer 45:889-895.

Wang RR, Li Y. 1998. In vitro evaluation of biocompatibility of experimental titanium alloys for dental restorations. J. Prosthet. Dent $80: 495-500$.

Wikipedia. 2014. Median income in Brazil by career in 2007. Available at: http://pt.wikipedia.org/wiki/Renda_no_Brasil. Accessed on July 2014.

Wingren G, Hallquist A, Hardell L. 1997. Diagnostic x-ray exposure and female papillary thyroid cancer: A pooled analysis of two Swedish studies. Eur. J. Cancer. Prev 6:550-556.

Zielinski JM, Garner MJ, Krewski D, Ashmore JP, Band PR, Fair ME, Jiang H, Letourneau EG, Semenciw R, Sont WN. 2005. Decreases in occupational exposure to ionizing radiation among Canadian dental workers. J. Can. Dent. Assoc 71:29-33.

\section{SUPPORTING INFORMATION}

Additional Supporting Information may be found in the online version of this article at the publisher's web site.

Disclosure Statement: The authors report no conflicts of interests.

${ }^{\mathrm{a}}$ Dr.Sergio Koifman died in May 2014. 